\title{
A Collaborative Medium for the Support of Conversational Props
}

\author{
Tom Brinck and Louis M. Gomez \\ Bellcore \\ 445 South St. \\ Morristown, NJ 07962 \\ hammer@bellcore.com, (201) 829-5238 \\ gomez@bellcore.com, (201) 829-4451
}

\begin{abstract}
Our work focuses on providing computational support for informal communication among people who are geographically separated. To better understand the use of artifacts in communication, we looked at the contents of office whiteboards after they had been used in conversations. Our analysis revealed that whiteboards are used to present and discuss various classes of objects with specific semantic properties. We call these objects "conversational props," and we have come to think of a whiteboard as a conversational medium in which props are introduced and manipulated. This study motivated our design of the Conversation Board, an experimental prototype of a multi-user drawing tool which allows remote use of conversational props. We compare the Conversation Board to various other multi-user drawing tools along a number of dimensions.
\end{abstract}

Keywords: design methodology, collaborative systems, group work, informal communication

\section{Introduction}

We have been exploring the design of multi-user applications to be used along with video communications systems, such as the CRUISER ${ }^{\mathrm{TM}}$ system [9][14]. The Cruiser system attempts to recreate a sense of informality common to workplace interactions among people. The intuition that video can add a new sense of presence to telecommunications stems from its ability to add facial expressions and gestures to standard audio-only conversations.

Recent observations of use of the CRUISER system [5] indicate that people find it to be a valuable

communications tool which enhances standard telephone communications. They also indicate that the CRUISER system is not currently a viable competitor to face-toface conversation. One of the reasons for this is that the CRUISER system does not allow people to easily share artifacts like drawings, graphs, or photographs, which are often part of face-to-face meetings. We have come to refer to these media as "conversational props". In the same sense as theatrical stage props, they add communicative realism to situations. In face-to-face conversation, almost anything can serve as a prop, from a napkin to a snapshot of the kids.

In the work reported here we explored the design and implementation of a shared "whiteboard", called the Conversation Board, that could make electronic props available for informal office communication when the participants are geographically separated. There are a number of domains in which this kind of capability for shared props is useful. For example, doctors could share $\mathrm{x}$-rays and medical illustratrions that are used during medical consulting. Electronic conversational props and desktop video could also enable new forms of classroom education over a distance where students not only see and hear their peers but have access to shared educational material.

To understand how geographically-separate office workers might use conversational props, we started by examining the use of office whiteboards as a medium for conversational props in face-to-face communication. We chose office whiteboards because they seemed to be commonly used in the support of conversation in the workplace. Our goal was not to build an electronic duplicate of a physical whiteboard, but instead to discover the communication intent that is supported by the office whiteboard and to use that information to inform the design of an electronic medium which supports the use of conversational props.

\section{The Study of Office Whiteboards \\ Procedure}

Our approach was to conduct spontaneous interviews of people in their offices about the current state of their whiteboards. Participants were asked to describe the sketches, writing, and diagrams that were currently on their boards and the conversations that led to them. This was entirely retrospective. We observed the whiteboard 
artifacts left behind after conversations had been completed.

The participants were 18 Bellcore employees. Ten were technical employees with backgrounds in mathematics, computer science, and electrical engineering. Eight were administrative employees who had financial and budgetary assignments. The interviews were conducted in their offices, and lasted 10 to 15 minutes.

Photographs were taken of the whiteboards which were later analyzed. The interviewer also made sketches of the whiteboard contents, annotating a sketch with information the participant supplied during the course of the interview.

\section{General Whiteboard Observations}

The 18 whiteboards we studied contained remnants of about 76 conversations. Of those, 17 conversations were on whiteboards of administrative participants, and the remaining 59 conversations were on whiteboards of technical participants.

Most whiteboard conversations involved two people, although four of the whiteboards contained entries which were generated only by a solitary user. Several people used their boards both alone and with others. Some reported that when they used the whiteboard alone it was to create objects which would later play a role in a meeting. Very few whiteboard conversations involved three or more people.

\section{Color}

All the whiteboards we observed contained multiple colors, as few as two and as many as five. This is perhaps not surprising, since whiteboard markers are sold in small sets of multiple colors. Some people used color to distinguish between different conversations whose scribblings were on the board at the same time. In this way, even if a board was crowded with a previous conversation which had used a black marker, people could avoid erasing the previous conversation and avoid confusing the two conversations by using a red marker for the new one. Others used color to distinguish between categories of information (e.g. home phone numbers versus work numbers) and to distinguish levels of detail.

\section{Persistence}

Seven of the people interviewed said that they would not want all or part of their whiteboards to be erased on the day of the interview. This is because people anticipated that they would find the props currently on the boards useful for subsequent conversations or for future reference. In addition, 17 of the 18 people reported that some aspect of the whiteboard had been or would be copied into some other more permanent medium, such as a computer file or viewgraph.

\section{Classes of Objects}

Our characterization of whiteboard objects had the explicit goal of determining how we might create software that supports the objects observed and maintains those semantic properties that an object has in conversational use. Each class of objects in Figure 1 has certain properties that people said they use when they introduced them into a conversation as props. For example, tables were sometimes used in the same way as spreadsheets. People reported that row/column sums in tables were modified dynamically throughout the course of meetings. From the perspective of our analysis, therefore, it was not sufficient to determine that a drawing tool could, with enough effort on the part of users, recreate the appearance of every object we observed. It was much more important to motivate the design and structure of an electronic whiteboard with features that captured the semantics of the objects that people used in whiteboard conversations.

With this purpose in mind, we totaled the number of objects occurring on the whiteboards within various categories. These totals are imperfect due to the uncertain recall of the interviewees as well as the occasional difficulty in clearly categorizing objects, but they capture the general trends. Figure 1 shows eight different kinds of objects that we were able to classify and their relative frequency of use by technical and administrative people. Since technical and administrative participants had different numbers of conversations represented on their boards, we chose not to present total counts of objects, but instead to present the average number of objects per conversation. Some of these objects and their use bear further explanation:

Text and lists. Figure 1 shows that an average conversation had about one text object (an average of .83 text objects per conversation for technical participants and .88 for non-technical participants). Text refers to objects that people appeared to treat as blocks of connected text, that is, short phrases, sentences, or paragraphs. Other instances of text, used simply to label things, were much too numerous to count. Since these were so numerous, it is clear that text was by far the most common kind of object found on a whiteboard. Many instances of text were serving the more specific conversational function of lists (based on, for instance, their linear and unconnected character), and so these were counted in a separate category. 
Geometric objects. Objects such as circles, lines, and rectangles are categorized as geometric pictures. Figure 1 shows that during a conversation involving administrative participants, an average of 1.4 geometric objects were drawn. Administrative participants used lines and boxes much more frequently than technical participants to separate and organize information. This accounts for much of the disparity between administrative and technical groups in this category.

Graphs. The objects labeled as graphs were, by and large, stylized representations of data. They were usually squiggles on unlabeled axes, which we presume were generated quickly to make a point and not to provide numerical accuracy. Graphs, block diagrams, and equations were used almost entirely by the study's technical participants.

Sketches. Objects were classified as sketches when it appeared that they could not easily be interpreted as belonging to a general category of structured object. For the most part, sketch objects appeared to be used for

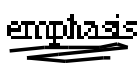

conversation.

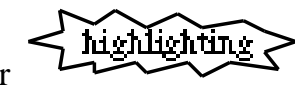

or as gestures in the

Other objects. The "Other" category in the figure was a collection of various other structured objects that people drew or imported to their boards. For example, two people had maps placed on their board, one of which was a floor plan. Other people used graphical trees to outline various relationships among objects. Two people also constructed layouts of computer screens.
Our analysis strongly suggests that whiteboards are not simply "drawing spaces" where people sketch forms devoid of intrinsic semantic content. Since intuitively we had viewed whiteboards primarily as a sketching medium, the most compelling aspect of the objects we observed was their rich semantic content. For instance, text was used more often than any other category. This is true despite the fact that whiteboards do not provide any specific facilitation for the use of text. The whiteboard marker merely facilitates sketching, but the way people use whiteboards suggests to us that they would prefer a medium that preserves the semantic structure of objects placed on the board. Physical whiteboards are sketch mediums because of the constraints provided by the physical environment, but electronic whiteboards need not simply provide sketch functionality as some previous ones have done. They should give people an easily-accessible and rich palette of objects that retain their structure once displayed and can be manipulated in terms of that structure.

Our observation indicates that text and geometric objects are props with wide applicability to both technical and administrative people. However, other props seem to have less generality. It is no surprise that different domains of work will require different kinds of conversational props. For instance, in the medical domain we expect that high-resolution images will be used quite frequently. In the design domain, we might expect the need for more sophisticated sketching capabilities. In the interface design domain, we would expect the use of menus, icons, and buttons.

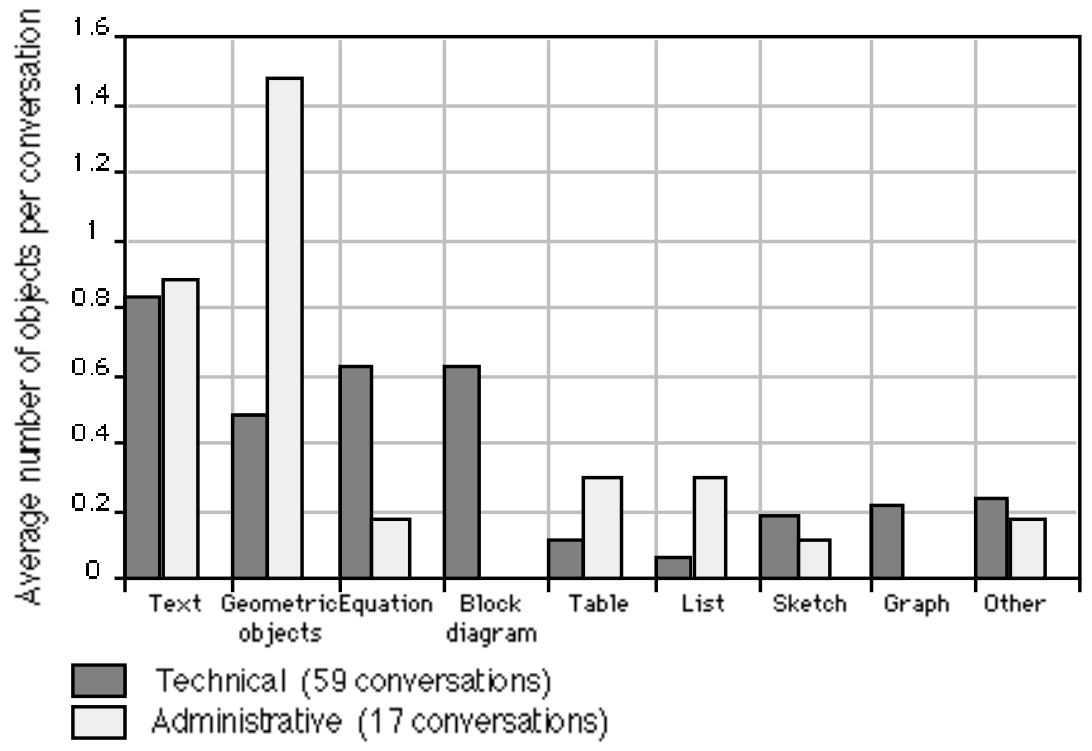

Figure 1. A categorization of the contents of office whiteboards for technical versus administrative users. 


\section{The Design of The Conversation Board}

From this study and the experience of other investigators (e.g. [3]) we formulated requirements for the Conversation Board. In this section, we describe our current prototype. Then we discuss how we approached our three central design goals: supporting structured props, supporting persistence, and providing fast highbandwidth communication, both between the users and between the users and the whiteboard.

\section{Our Prototype}

The Conversation Board is a structured graphics editor with a palette of tools and a canvas which serves as the shared conversation space. The structured graphics are similar in style to MacDraw ${ }^{\mathrm{TM}}$. However, unlike MacDraw, the Conversation Board is not intended to support the creation of high-quality drawings, but to facilitate the conversational use of objects.

The Conversation Board is being developed in the RENDEZVOUS ${ }^{\mathrm{TM}}$ system, an experimental toolkit designed at Bellcore to support multi-user interfaces [7]. The RENDEZVOUS language is built on top of Common Lisp and uses the $\mathrm{X}$ Window ${ }^{\mathrm{TM}}$ system as its terminal model. The Conversation Board prototype has been used informally within our lab. New users have adapted very quickly to its interface. The current version of the Conversation Board has tools for multi-user sketching with various colored markers, editable multiline text, circles, rectangles, lines, connectors, and the ability to import images and sound clips. It includes features such as telepointers, saving and loading, undo, and cut $\&$ paste.

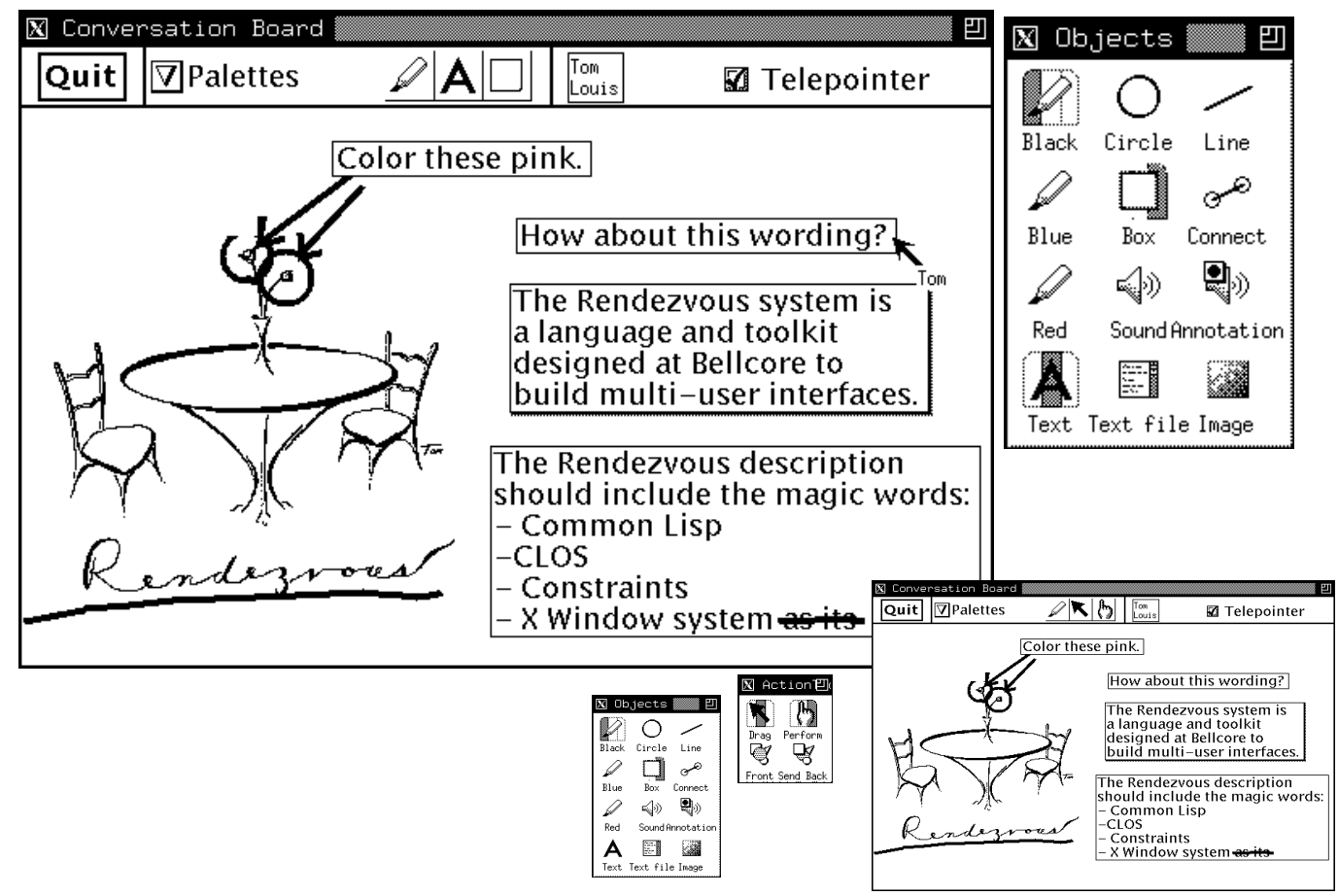

Figure 2. This shows a view of the Conversation Board for one user and a miniature of the view of a second user. It is being used to brainstorm a description of the Rendezvous system. The main window consists of a control region at the top and a canvas where all the drawing occurs. There are several tool palettes available. The main one is shown at the top right.

\section{Structured Props}

The fundamental lesson we obtained from our study is that there are common categories of objects on office whiteboards with a structure that reflects how they can be manipulated in conversational use. We provide a tool palette of basic "drawing" tools for access to the most common kinds of objects which we observed on office whiteboards. Since users can edit these structured objects, they can manipulate the props with the semantic operations that are conceptually appropriate. These props can be created on the canvas as complete units and then moved, edited, and deleted independently. Figure 2 shows the Conversation Board's palette and canvas.

Sketches. For sketches that couldn't be classified as other structures, we chose to provide markers of 3 
different colors. This allows color to be used much as it was on physical whiteboards. The markers will also be appropriate for creating graphs that show general shapes of curves but not numerical data.

Geometric objects. Oval, line, arrow, and rectangle tools should be sufficient to support most cases of geometric pictures we observed.

Text, lists, and equations. A tool for editable text supports these three categories. Because text was quite frequently used for simple labeling, the text tool should facilitate short, one-line pieces of text. This clearly won't be appropriate for all types of equations - in the general case the sketch tool may be necessary for supporting equations. Providing any tool to support a broad range of math notation poses a considerable design challenge if it must also support rapid communication.

Tables. A simple table tool would be sufficient for duplicating the use of a table on a whiteboard, although we suspect that office users would ask for more and more spreadsheet functionality in this tool, since the ones who use tables on their whiteboards are often the same people who use spreadsheets in their work and would want to transfer data between the two forms.

Connectors. To support the use of block diagrams, we provide a connector tool that draws a line or arrow between two other objects and remains connected to them even when the objects are moved. The block diagrams we observed contained a wide variety of different nodes, drawn as circles, rectangles, text, or more complicated objects. They were also labeled in a wide variety of ways with text. The one structural aspect they had in common was the use of connecting lines and arrows that stay attached to the objects they link, even if those objects are moved. This behavior is reasonably well captured with a connector tool.

Images. Hand-drawn pictures of real-world objects were rarely observed on whiteboards, though we saw cases in which photographs and maps were placed on whiteboards. We believe one reason for this is that people can't draw pictures in a sufficiently timely fashion to support a rapidly evolving conversation. For this reason, we provide an on-line library of pictures that can be brought into the whiteboard. Our current library contains, for example, maps and personal photographs. We would also like to have a scanner readily available for adding images, although it would have to work quickly in order to mesh with the flow of the conversation.

\section{Object Persistence}

Many users leave the contents of their whiteboards unerased for long periods of time just in case they feel a need to reuse their previous sketches. These results imply a need for users of the Conversation Board to be able to save and retrieve the contents of their whiteboards. Many users need to copy their tables into spreadsheet files or their text to text files. It may also be useful to save the entire conversational context - the date and time of the conversation and any associated audio or video communication.

\section{Fast, High-Bandwidth Communication}

Here we describe a few approaches to improving the speed and the bandwidth of communication.

Optimized tool access. As we noted, central to our design philosophy has been the support of spontaneous, informal conversation. But there exists a tension between improving the rapidity of whiteboard interaction by providing specialized structured tools and the need to provide a minimal toolset for simplicity of interaction. A design providing only a simple sketch tool might, for example, improve spontaneity by decreasing the range of alternatives the user must consider and removing the time necessary to switch between tools.

Given the advantages of editable structures, we feel it would be a mistake to be too austere in the choice of tools an application supports. Instead, we suggest that there should be user interface accelerations to move between tools. One idea we are trying in our current implementation of the Conversation Board is customizing the use of a three-button mouse. If we had only 3 tools, we could use each mouse button to perform the operations of a single tool. However, there are many more tools available than mouse buttons. With a one-button mouse, a single tool is selected from the numerous possible tools available in a tool palette. Our design extends this notion so that there is a tool selected from the palette for each button of the threebutton mouse. Instead of just inverting the chosen tool in the palette, the selected tools have a picture highlighting the left, middle, or right mouse buttons to indicate the mouse buttons with which the tools are associated (see Figure 2, where the black marker is assigned to the left mouse button, the text tool to the middle, and the rectangle tool to the right). Each Conversation Board user can make the tools that are currently most valuable to the conversation the easiest to access.

We believe that this will be no more difficult to use than a one-button mouse, since this tool palette functions the 
same as a one-button mouse as long as the user only chooses to use one mouse button. However, this gives an advantage over a one-button mouse in that it gives the user the ability to use a larger "working set" of tools, thus reducing the amount of time spent switching tools.

Gesturing. Gesturing is something that is often done in the context of a face-to-face conversation [3], but can be difficult when the prop is no longer something people can physically point to. People often used sketching in order to gesture. We believe that in many cases a telepointer can be used to support the same purpose. A telepointer (used in several other systems, e.g. [11]) displays to users the screen pointers of the other users with whom they are interacting. Telepointers widen the bandwidth of communication between users by conveying information without creating further work for them. However, when telepointers convey irrelevant or distracting information or otherwise just get in the way, the Conversation Board provides a checkbox to turn off a users telepointer. Other systems (e.g. Commune [12]) always provide a telepointer. At least one (WScrawl [19]) provides the telepointer as a tool, thus making it impossible to gesture and draw simultaneously.

Simultaneous Work. The ability to work simultaneously on a shared surface helps people to communicate more quickly since more work can be done in parallel. Bly [3] observed people interacting in design sessions and indicated that most drawing events occurred on regions of the drawing that were shared. We would like to support this, and also allow users to work at the same time on the same region. Therefore, the Conversation Board canvas is shared, but control is not. Different users have the ability to select different tools, select different objects, and perform different commands at the same time. In addition, their views of the whiteboard contents may need to be customized. For instance, when working on a large piece of text, each user may want to scroll to different portions of the text to work on it.

\section{RELATED WORK}

Our goal has been the creation of an electronic conversation space, where a conversation space is a space into which props are introduced and manipulated. We use the following set of issues to distinguish between other systems that are similar. Each of these will be discussed in turn.

\section{Drawing characteristics}

Structured vs. bitmap graphics
Level of functionality

Speed vs. quality

Degree of specialization

\section{Multi-user characteristics}

Number of users

Custom vs. duplicated views

Multi-user awareness

Simultaneous vs. token-passing interaction

Same vs. different place

Same vs. different time

\section{Structured vs. bitmap graphics}

The Conversation Board is a structured graphics tool after objects are placed on the shared canvas they can still be edited and moved. Xsketch [11] is another shared structured drawing tool. It supports polyline, box, and text objects, and allows some limited editing capabilities. The NeXT Greyboard is a mutli-user structured graphics editor built on top of the NeXT Draw program. In addition to supporting standard structured drawing tools, it allows users to drop in arbitrary files which are shipped and opened on each users system. A number of electronic whiteboards use bitmap graphics. These whiteboards more closely emulate the model of a physical whiteboard. They primarily focus on supporting sketching functionality, and often also support erasure, uneditable text, and telepointers. These tools include BoardNoter [16], WScrawl [19], WE-MET [20], Commune [12], and GroupSketch [6].

\section{Level of functionality}

An important tradeoff is involved in deciding how many capabilities to provide a user. With fewer capabilities, there is less for a user to learn and fewer decisions to be made. But each new capability can, once learned, make the situations it was designed for easier. This tradeoff is especially important in conversational use. If the tools take too much time to learn, they either won't be used or will disrupt the conversation.

A physical whiteboard has basically two operations available at any time - draw or erase. Though a physical whiteboard has very limited functionality, these tools are nevertheless powerful enough to create complex imagery (with sufficient time) and are very simple to use. Systems such as BoardNoter and GroupSketch, which in many ways try to emulate the functionality of physical whiteboards using bitmap graphics and simple markers (though they also support text entry), seem to be successful at achieving the spontaneity and ease-ofuse that physical whiteboards embody.

The Conversation Board provides many more tools than 
these systems. Another application which provides more functionality is WScrawl, which has tools such as text, line, and box, and the ability to read in a bitmap from a file. There is a careful balance that must be considered when determining level of functionality. For instance, our study indicated that text would be used so often that it seemed worth the time it takes to learn to use it. On the other hand, the connector tool may just be another distraction for administrative users who have no need to draw block diagrams.

\section{Speed vs. Quality}

We chose to build a tool that would be used in conversation, therefore the emphasis of the Conversation Board is rapid and unobtrusive construction of a shared drawing, rather than the production of high-quality imagery. Some applications of shared drawing surfaces may require very polished results, such as the cooperative design of a logo or advertisement. One approach to producing high-quality results is to share a high-end single-user graphics editor with a windowsharing system, such as Dialogo [10], Shadows [13], or Rapport [1], or a screen-sharing system such as Timbuktu $^{\mathrm{TM}}$ [4]. For instance, the Timbuktu software allows an application like MacDraw to be shared among multiple users. However, a high-end single-user graphics editor will not always be appropriate in general communication situations. The use of an office whiteboard is an excellent example where high-quality is not as important as the communicative value of rapidly expressing ideas.

Rulers and the ability to precisely adjust pixel locations and sizes of objects are examples of features the Conversation Board willingly omitted under the assumption that this simplified the interface and that these features which enabled enhanced quality of resulting images were not critical to the ability to communicate effectively. Of course, speed and quality do not always have to be conflicting goals. For instance, by providing an invisible grid, we could make it easy for users to align objects without slowing down the interaction.

\section{Degree of specialization}

SharedARK [15] is an example of a system aimed at very specific types of interactions, shared physics problem-solving. Systems such as the Conversation Board or WScrawl are designed with very general-purpose tools, such as markers and text, which have very little behavior associated with them.

\section{Number of users}

Our study of whiteboard use indicated that typical office conversations involving a whiteboard had only 2 or 3 participants. It is unclear whether this will remain true for electronic boards since physical interference between people will no longer be a problem in using the board. Though it wasn't a specific design goal, the Conversation Board is built to support any number of users - any limitation on the number of users is due to limited computational power and a need for good response time.

One set of electronic whiteboards incorporate video into the shared design space. These systems have difficulty supporting more than 2 or 3 users simultaneously since overlaying multiple videos can be confusing and results in image degradation. VideoDraw [17] provides a small workspace onto which users can draw with actual markers, and shows video of the hands of the users as they work on their sketches. TeamWorkStation [8] is similar to VideoDraw but also allows video overlays of computer windows. This enables users to draw by hand or with any computer drawing package.

VideoWhiteboard [18] is a wall-mounted whiteboard which portrays a shadow of the other user on the surface of the board. These systems are unique in that they can convey a class of gesture and personal expression that is extremely difficult to capture in a whiteboard surface lacking video. They can also incorporate physical props into the shared workspace. However, these systems require specialized hardware and the interaction on these systems is limited by the fact that it is not possible for users to modify the work of their conversational partners.

\section{Custom vs. duplicated views}

Several parts of the Conversation Board interface are customized to each user. Only the canvas is shared identically between all users. Users can have different tools selected at the same time. Thus, one user can be drawing with a red marker, another with a blue marker, and another creating text, all simultaneously. Sharedwindow systems allow single-user applications to be shared by multiple users with completely duplicated views. Since single-user applications are written without any knowledge of multiple people, they cannot control how much information will be shared between users (all of it will). For instance, using MacDraw with Timbuktu gives users the last tool that anyone selected, since the tool selection is duplicated.

\section{Multi-user awareness}

Applications that are written specifically for multiple users can be multi-user aware - they can provide customized views and provide information about the conference itself and the roles of the various participants. 
Single-user applications running under shared windowing systems were not written with the anticipation of multiple users and therefore can't provide these features. For instance, they could not provide users with a list of the current conference participants. Shared windowing systems typically provide conference information and telepointers, but the application cannot use this information.

Simultaneous vs. token-passing interaction Some multi-user drawing programs, such as the NeXT Greyboard, require users to pass a token (the "marker") between them. The user who currently holds the marker gets to draw. The other users can watch, but cannot interact with the drawing. This method can be easier to implement because user inputs don't need to be synchronized - only one user can draw at a time, and the actions of the one user are broadcast to the other users. The quality of interaction is similar to that of a physical whiteboard, where typically only one person draws at once.

Many multi-user drawing surfaces allow the users to draw simultaneously as the Conversation Board does, including for instance, VideoDraw and WScrawl. Bly [3] and Whittaker et al [19] both give evidence that this may be preferred in conversation.

\section{Same vs. different time}

We started our design with the assumption that our tool would support conversation at a distance. Thus, we assumed that these interactions would occur at the same time and in different places. All of the above systems are designed for synchronous interaction. Any system, such as the NeXT Greyboard, which allow the contents of the interaction to be saved and later retrieved, can to some extent be used asynchronously.

\section{Same vs. different place}

Most of the systems mentioned have been designed for users who are viewing the interaction on separate monitors - these tools can be colocated but typically are not. Boardnoter [16] was designed for use in a single meeting room. MMM [2] is a collaborative environment for multi-user editors, such as a text editor and a rectangle editor, that is explicitly designed for users sharing a single monitor.

\section{Conclusion}

The dimensions of comparison between multi-user drawing systems that we've described enables us to distinguish among systems which seem to serve very similar goals. However, the choices made along each of these dimensions depend on the particular way each system is intended to be used. We made our choices based on our goal of supporting informal conversation.

Our study of office whiteboards was motivated by the desire to learn the gross characteristics of the use of props in conversation. This analysis drove our choice of providing structured objects and deciding which types of structured objects to provide and what their behavior should be.

The research reported here has established the value of structured props to conversation. It has also given us initial design guidelines for artifacts that may enhance communication by supporting the straightforward introduction of props into informal conversation.

\section{Acknowledgements}

We wish to thank the other members of the RENDEZVOUS team - Ralph Hill, John Patterson, Steve Rohall, and Wayne Wilner - for their many contributions to both this paper and the work itself. We also thank Scott Stornetta and Will Hill for valuable comments on an earlier version of this paper.

\section{$\underline{\text { References }}$}

1. Ahuja, S.R., Ensor, J.R., \& Lucco, S.E. A comparison of application sharing mechanisms in realtime desktop conferencing systems, in Proc. COIS '90 Conference on Office Information Systems (Cambridge, MA, Apr. 25-27). ACM, New York, 1990, pp. 238248 .

2. Bier, E.A. \& Freeman, S. MMM: A user interface architecture for shared editors on a single screen, in Proc. UIST '91 Symposium on User Interface Software and Technology (Hilton Head, SC, Nov. 1113). ACM, New York, 1991, pp. 79-86.

3. Bly, S.A. A use of drawing surfaces in different collaborative settings, in Proc. CSCW ' 88 Conference on Computer-Supported Cooperative Work (Portland Oregon, Sep. 26-28). ACM, New York, 1988, pp. 250-256.

4. Farallon Timbuktu User's Guide. Farallon Computing, Inc. Berkeley, CA, 1988.

5. Fish, R.S., Kraut, R.E., Root, R.W. \& Rice, R.E. Evaluating video as a technology for informal communication, in Proc. CHI '92. ACM, New York, 1992.

6. Greenberg, S. \& Bohnet, R. GroupSketch: a 
multi-user sketchpad for geographically-distributed small groups, in Proc. Graphics Interface '91 (Toronto, June 37). Canadian Information Processing Society, Toronto, 1991, pp. 207-215.

7. Hill, R.D. A 2-D graphics system for multiuser interactive graphics based on objects and constraints, in E. H. Blake \& P. Wisskirchen (Eds.) Advances in Object-Oriented Graphics I. SpringerVerlag, New York, 1991.

8. Ishii, H. TeamWorkStation: towards a seamless shared workspace, in Proc. CSCW '90 Conference on Computer-Supported Cooperative Work (Los Angeles, CA., October 7-10). ACM, New York, 1990, pp. 13-26.

9. Kraut, R.E., Fish, R.S., Root, R.W., \& Chalfonte, B.L. Informal communication in organizations: form, function, and technology, in S. Oskamp \& S. Spacapan (eds.), Human reactions to technology: the Claremont symposium on applied social psychology. Sage Publications, Beverly Hills, 1990.

10. Lantz, K. An experiment in integrating multimedia conferencing, in Proc. CSCW ' 86 Conference on Computer-Supported Cooperative Work (Austin, TX, December). ACM, New York, 1986, pp. 267-275.

11. Lee, J.J. Xsketch: a multi-user sketching tool for X11, in Proc. COIS '90 Conference on Office Information Systems (Cambridge, MA., April 25-27). ACM, New York, 1990, pp.169-173.

12. Minneman, S.L. \& Bly, S.A. Managing à trois: a study of a multi-user drawing tool in distributed design work, in Proc. CHI '91 Human Factors in Computing Systems (New Orleans, LA., April 27 May 2). ACM, New York, 1991, pp. 217-224.

13. Patterson, J.F. The good, the bad, and the ugly of window sharing in $\mathrm{X}$, in Proc. the Fourth Annual $\mathrm{X}$ Technical Conference (Boston, MA, January 15-17). 1990.

14. Root, R.W. Design of a multi-media vehicle for social browsing, in Proc. CSCW ' 88 Conference on Computer-Supported Cooperative Work (Portland Oregon, September 26-28). ACM, New York, 1988, pp. 25-38.

15. Smith, R. B., O'Shea, T., O'Malley, C., Scanlon, E., Taylor, J. Preliminary experiments with a distributed, multi-media, problem solving environment, in Proc. EC-CSCW '89 1st European Conference on Computer-Supported Cooperative Work (Gatwick, U.K., September 13-15). Computer Sciences House, Slough, U.K., 1989, pp 19-34.

16. Stefik, M., Foster, G., Bobrow, D.G., Kahn, K., Lanning, S., \& Suchman, L. Beyond the chalkboard: computer support for collaboration and problem solving in meetings. Communications of the ACM 30,1 (1987), pp. 32-47.

17. Tang, J.C. \& Minneman, S.L. VideoDraw: a video interface for collaborative drawing, in Proc. CHI '90 Human Factors in Computing Systems (Seattle, WA., April 1-5). ACM, New York, 1990, pp. 313-320.

18. Tang, J.C. \& Minneman, S.L.

VideoWhiteboard: video shadows to support remote collaboration, in Proc. CHI '91 Human Factors in Computing Systems (New Orleans, LA., April 27 May 2), ACM, New York, 1991, pp. 315-322.

19. Whittaker, S., Brennan, S.E., \& Clark, H.H. Co-ordinating activity: an analysis of interaction in computer-supported co-operative work, in Proc. CHI '91 Human Factors in Computing Systems (New Orleans, LA., April 27 - May 2). ACM, New York, 1991, pp. 361-367.

20. Wolf, C.G., Rhyne, J.R., Zorman, L.A., \& Ossher, H.L. WE-MET (Window Environment-Meeting Enhancement Tools), in Proc. CHI '91 Human Factors in Computing Systems (New Orleans, LA., April 27 May 2). ACM, New York, 1991, pp. 441-442. 